\title{
Research of Adaptive Fault-Tolerant Control Based on T-S Fuzzy Model
}

\author{
Z. J. Yu and Y. N. Xu
}

\begin{abstract}
The adaptive fault-tolerant control problem for descriptor nonlinear system is researched in this paper. Contents include the properties of Adaptive Fault-tolerant like uncertainties, state delays, and input delays using T-S fuzzy model. First, the design of the state observer to estimate system state and sensor fault is introduced. Then, the adaptive fault-tolerant control structure with the state observer is proposed when combining with the sliding model control (SMC) and the adaptive generic model control (AGMC). Finally, an adaptive fault-tolerant control structure for nonlinear descriptor system is constructed. About above results, both of theoretical analysis and illustrative examples demonstrating the feasibility and validity were proposed.
\end{abstract}

Index Terms-Fault-tolerant control, T-S fuzzy, observer, sliding model control, adaptive generic model control.

\section{INTRODUCTION}

Because of an effective method to improve the reliability and safety of devices adaptive fault-tolerant control technology has been widely investigated by industries [1]-[3]. Recently, researches on fault-tolerant control were developed rapidly using fuzzy system, neural network, expert system, pattern recognition, and other methods. Among them, T-S fuzzy model was proposed by Takagi and Sugeno to described complex nonlinear systems. Furthermore, Taniguichi T et al., extended this normal T-S fuzzy model to generalized situations [4]. T-S fuzzy model was proposed to describe the complicated nonlinear system, though the reliability of a nonlinear descriptor system and the optimal fault-tolerant control were not taken into consideration [5]-[7]. Z. W. Gao proposed a descriptor system, describe the fuzzy system with sensor fault [8]. Designed fuzzy state observer can estimate both the system states and faults simultaneously. The problem is that the system time-delay was ignored. These nonlinear adaptive fault-tolerant systems are usually designed based on nonlinear process model and nonlinear controllers. One of them is the generic control model (GMC) [9] which was proposed in 1988 introduced the new control structure in which the nonlinear process model can be used directly in control strategy. GMC has been applied experimentally to a diverse range of processes, such as bench-scale yeast fermentation, binary distillation, and a continuous metallurgical process. But GMC has unobvious

Manuscript received November 18, 2013; revised January 10, 2014. This work was supported by the National Natural Science Foundation of China (61361003) and Jilin University of State Key Laboratory of Automotive Simulation and Control open fund (20121120).

The authors are with the division of electronic and communication engineering of Yanbian University, Yanji, China (e-mail: 2013050243@ybu.edu.cn,ynxu@ybu.edu.cn). physical meaning and not particularly good effect [10]. P. D. Signal proposed general model adaptive control which using model parameters adaptive changing to estimate the model parameters online. The weakness of Kalman filter is a poor robustness to model errors [11]. Sliding model control (SMC) is an effective nonlinear control method which has attractive features such as a fast response, a good transient performance, and the insensitiveness of uncertainties and external disturbances. It can improve the reliability and robustness of the system [12]-[14]. Therefore, it is proposed in this paper that the Kalman filter in general model adaptive control method can be replaced by the state observer, the general model adaptive fault-tolerant control architecture can be proposed based on SMC control.

This paper is organized as follows. In Section II, a class of descriptor nonlinear system with time-delays, and uncertainties are described by T-S fuzzy model. Then, the state observer is designed which estimates system state and sensor fault. In Section III, the adaptive general model control based on sliding model control is proposed by combine SMC and AGMC to improve the robustness and effectiveness of the nonlinear descriptor system. In Section $\mathrm{IV}$, the control law reconfiguration is proposed in case of sensor fault occurring. In Section V, the whole adaptive fault-tolerant control structure for nonlinear system was proposed based on T-S fuzzy model. Finally, both the theoretical analysis and illustrative example demonstrate the feasibility and validity of the adaptive fault-tolerant control structure were proposed.

\section{T-S FUZZY DESCRIPTOR SYSTEM MODEL}

T-S fuzzy [15]-[17] descriptor system is a nonlinear system which is described by a group of if-then $(i-t h)$ fuzzy rules, and each rule represents one subsystem. Considering the uncertain model of T-S fuzzy descriptor system with state delays and input delays, the $i$-th fuzzy rule can be described as follows:

$$
\begin{aligned}
& R_{i}: \text { If } \xi_{1}(t) \text { is } M_{1 i} \text { and } \xi_{p}(t) \text { is } M_{p i} \text {, then } \\
& \begin{aligned}
\dot{x}(t) & =\left[A_{i}+\Delta A_{i}(t)\right] x(t)+\left[A_{i d}+\Delta A_{i d}(t)\right] x(t-d) \\
& +\left[B_{i}+\Delta B_{i}(t)\right] u(t)+\left[B_{i d}+\Delta B_{i d}(t)\right] u(t-\tau)
\end{aligned} \\
& y(t)=C x(t), \quad i=1,2, \cdots, r
\end{aligned}
$$

Here, $\xi_{1}(t) \cdots \xi_{p}(t)$ are the premise variables assumed measurable, $M_{1 i} \cdots M_{p i}$ are the fuzzy sets. $r$ is the number of fuzzy rules. $x(t) \in R^{n}$ is the state vector. $u(t) \in R^{n}$ is the input vector. $y(t) \in R^{n}$ is the measurable output vector. $d$ is the 
state time-delay and $\tau$ is the input time-delay. $A_{i}, A_{i d}, B_{i}, B_{i d}, C_{i}$ and $N_{i}$ are the known constant matrices with appropriate dimensions. $\Delta A_{i}(t), \Delta A_{i d}(t), \Delta B_{i}(t)$ and $\Delta B_{i d}(t)$ are the parametric uncertainties and considered norm-bounded, in the form: $\left[\begin{array}{llll}\Delta A_{i} & \Delta A_{i d} & \Delta B_{i} & \Delta B_{i d}\end{array}\right]=H_{i} F_{i}(t)$

$\left[\begin{array}{llll}E_{1 i} & E_{2 i} & E_{3 i} & E_{4 i}\end{array}\right]$. Where $H_{i}, E_{1 i}, E_{2 i}, E_{3 i}$ and $E_{4 i}$ are the known real constant matrices with appropriate dimension. $F_{i}(t)$ is an unknown matrix function satisfying $F_{i}(t)^{T} F_{i}(t) \leq I$, in where $I$ is the identity matrix of appropriate dimension.

Using center-average defuzzifier, product interference, and singleton fuzzifier, the T-S fuzzy system can be inferred asfollows:

$$
\begin{aligned}
& \dot{x}(t)=\sum_{i=1}^{r} q_{i}(\xi(t)) \cdot\left\{\begin{array}{l}
{\left[A_{i}+\Delta A_{i}(t)\right] x(t)+\left[B_{i}+\Delta B_{i}(t)\right] u(t)} \\
+\left[A_{i d}+\Delta A_{i d}(t)\right] x(t-d) \\
+\left[B_{i d}+\Delta B_{i d}(t)\right] u(t-\tau)
\end{array}\right\} \\
& y(t)=\sum_{i=1}^{r} q_{i}(\xi(t)) C_{i} x(t), i=1,2, \ldots, r
\end{aligned}
$$

where the rules are defined as follows:

$$
q_{i}(\xi(t))=\frac{w_{i}(\xi(t))}{\sum_{i=1}^{r} w_{i}(\xi(t))}, \quad w_{i}(\xi(t))=\prod_{j=1}^{p} M_{l}^{i}(\xi(t)), \sum_{i=1}^{r} q_{i}(\xi(t))=1
$$

Considering the state of the system (1) is immeasurable, we construct an observer which can estimate both the system state and sensor fault directly. If the delay parameters have been known accurately, an observer contains time-delay parameters in the original system. In this paper, because accurate parameters in practical systems can't be got, we constructed a state observer without time-delay parameters in the original system.

We established the observer which can estimate both the system state and sensor fault as follows:

$$
\begin{aligned}
& E_{n} \varphi(t)=\sum_{i=1}^{r} q_{i}(\xi(t))\left[\bar{A}_{n i} \varphi(t)+\bar{B}_{i} u(t)\right] \\
& \hat{\bar{x}}(t)=\varphi(t)+H y(t)
\end{aligned}
$$

where $\varphi(t)$ is an auxiliary vector of the observer and $\hat{\bar{x}}(t)$ is the state estimation of (1). Obviously, $\hat{\bar{x}}(t)$ contains the estimation of state. $H$ is the observer gain to be determined later.

To obtain the parameters of the state observer, we defined the estimation error as $\bar{e}(t)=\bar{x}(t)-\hat{\bar{x}}(t)$. From equation (2) and equation (3), we obtain,

$$
E_{n} \dot{\bar{e}}(t)=\sum_{i=1}^{r} q_{i}(\xi(t))\left\{\begin{array}{l}
A_{n i} \bar{e}(t)+\left(\bar{A}_{i d}+\Delta \bar{A}_{i d}\right) x(t-d) \\
+\left(\Delta \bar{B}_{i}+\Delta \bar{B}_{i d}\right) \mu(t-\tau)+\Delta \bar{A}_{i} x(t)
\end{array}\right\}
$$

Then, it is calculated as following result:

$$
A_{n i}=\left[\begin{array}{cc}
A_{i} & 0 \\
-C & -I
\end{array}\right], E_{n}=\left[\begin{array}{cc}
I & 0 \\
C & I
\end{array}\right], H=\left(\begin{array}{l}
0 \\
I
\end{array}\right)
$$

\section{AdAPtive General Model CONTROL BASED ON SLIDING MODEL CONTROL (SMC-AGMC)}

In order to use adaptive general model control, the nonlinear system (1) with multiple inputs and outputs is transferred as follows:

$$
\begin{aligned}
& x(t)=f(x)+g(x) u(t) \\
& y(t)=h x(t)+y_{s}(t)
\end{aligned}
$$

where, $f(x), g(x)$ and $h(x)$ are the nonlinear functions of $x(t) \cdot y_{s}(t)$ is the sensor fault vector, and every relative order of outputs is 1 . In classical optimal control, the trajectory of output $y(t)$ is usually compared with some nominal trajectory as a system performance index. Having considered the speediness and unbiased property of the system, in order to get the satisfied output response, the reference trajectory of output is defined as follows:

$$
\begin{aligned}
y_{r}(t) & =y^{(n)}(t)+k_{r 0} y^{(n-1)}(t)+\cdots+k_{2} \dot{y}(t) \\
& =K_{1}\left[y_{r}(t)-y_{s p}(t)\right]+K_{2} \int_{0}^{t}\left[y_{r}(t)-y_{s p}(t)\right] d t
\end{aligned}
$$

where $y_{s p}(t)$ is the output setting. By choosing the matrix $K_{1}$ and $K_{2}$, the reference trajectory can be improved to the satisfied output response. By choosing control input $u(t)$, the system output can be made to track the reference trajectory as much as possible.

From equation (6), it can be calculated that:

$$
\begin{aligned}
y^{(n)}(t) & =K_{1}\left[y_{r}(t)-y_{s p}(t)\right]+K_{2} \int_{0}^{t}\left[y_{r}(t)-y_{s p}(t)\right] d t \\
& -k_{r 0} y^{(n-1)}(t)-\cdots-k_{2} \dot{y}(t)
\end{aligned}
$$

SMC is one of the effective robust control methods for nonlinear system; it provides system dynamics with an invariance property to uncertainties. This paper combines SMC and AGMC to improve the robustness of the adaptive general model control. Let the model tracking error in SMC be defined as $e_{y 1}(t)=y(t)-y_{r}(t)$, that is same to $\dot{e}_{y 1}(t)=\dot{y}(t)-\dot{y}_{r}(t)$. Then, the error matrix could be defined as $E_{1}=\left[e_{y 1}(t) \dot{e}_{y 1}(t)\right]^{T}$.

Variable structural control requires a sliding model with ideal sliding modality, good dynamic quality, and robustness. All of them can be achieved by appropriate choice of the sliding surface model that is designed by closed-loop performance in the state of a variable space. Then, design the control scheme, so that the system trajectories are forced toward the sliding surface and stay on it. Therefore, the sliding surface in SMC-AGMC can be designed as:

$$
s_{1}(t)=k_{1} e_{y 1}(t)+k_{2} \int_{0}^{t} e_{y 1}(\tau) d \tau
$$

According to sliding control method, design the general sliding model reaching law as $\dot{s}_{1}(t)=-\rho \operatorname{sgn}\left(s_{1}(t)\right)$. Then based on equation (8) can get:

$$
\dot{s}_{1}(t)=k_{1} \dot{e}_{y 1}(t)+k_{2} e_{y 1}(t)
$$


In order to get the sliding control law, transfer equation (9) as:

$$
\begin{aligned}
\dot{s}_{1}(t) & =k_{1} \dot{e}_{y 1}(t)+k_{2}\left[y(t)-y_{r}(t)\right] \\
& =k_{1} \dot{e}_{y 1}(t)-k_{2} y_{r}(t)+k_{2} C_{i}\left(f(x)+g(x) u_{1}(t)\right) \\
& =-\rho \operatorname{sgn}\left(s_{1}(t)\right)
\end{aligned}
$$

Finally, the sliding control law is obtained as:

$$
u_{1}(t)=\frac{k_{2} y_{r}(t)-k_{1} \dot{e}_{y 1}(t)-\rho \operatorname{sgn}\left(s_{1}(t)\right)-k_{2} C_{i} f(x)}{k_{2} C_{i} g(x)}
$$

The stability analysis of the SMC-AGMC proofed as follows:

$\dot{s}_{1}(t)=-\rho \operatorname{sgn}\left(s_{1}(t)\right)$ and $V=1 / 2 s_{1}^{2}(t)$ are obtained from equation (10) and Lyapunov function, respectively. As a result, the differentiating $V$ and time yields following equation,

$$
\begin{aligned}
\dot{V} & =s_{1}(t) \dot{s}_{1}(t) \\
& =s_{1}(t)\left[-\rho \operatorname{sgn}\left(s_{1}(t)\right)\right] \\
& =-\rho\left|s_{1}(t)\right| \\
& \leq 0
\end{aligned}
$$

Similarly, the system output $y(t)$ is ineffective when the sensor fault is detected. In this case, replace $y(t)$ in SMC-AGMC by the estimated output $\hat{y}(t)$ which is got by state observer. Then get the method of state estimation and output prediction based on SMC-AGMC. According to sliding model control method the model tracking error is can be defined as $e_{y 2}(t)=\hat{y}(t)-y_{r}(t)$ this means $\dot{e}_{y 2}(t)=\dot{\hat{y}}(t)-\dot{y}_{r}(t)$ also. The error matrix can be defined as $E_{2}=\left[e_{y 2}(t) \dot{e}_{y 2}(t)\right]^{T}$. The sliding surface in term of $e_{y 2}(t)$ is $s_{2}(t)=k_{1} e_{y 2}(t)+k_{2} \int_{0}^{t} e_{y 2}(\tau) d \tau$. Therefore, the sliding control law is designed as:

$$
u_{2}=\frac{k_{2} y_{r}(t)-k_{1} \dot{e}_{y 2}(t)-\rho \operatorname{sgn}\left(s_{2}(t)\right)-k_{2} C_{i} f(x)}{k_{2} C_{i} g(x)}
$$

In the case of detecting fault, the proof of the stability by the sliding model control method is same to equation (12).

\section{The CONTROL LAw ReCONFIGURATION LOGIC}

The active fault-tolerant control system should have an algorithm for fault estimation and isolation for unknown faults. The isolation of the system fault is performed based on adjusting the state observer estimation and the sensor fault. Fig. 1 shows a control law reconfiguration when sensor fault occurs.

First, estimate the sensor fault vector $y_{s}(t)$ by state observer. Then, use the estimation value $\hat{y}_{s}(t)$ to construct the fault detection and isolation algorithm. Finally, the control law reconfiguration logic is constructed as shown in
Fig. 1. As a result, the corresponding fault-tolerant logic will be gotten when the sensor fault is detected. In Fig. 1, $\varepsilon_{1}$ and $\varepsilon_{2}$ are threshold values defined before. If the condition satisfies $\left|\hat{y}_{s}(t)\right|>\varepsilon_{1}$, it will be considered that the sensor has fault. Then, the control law will be switched to the state estimation and output prediction of SMC-AGMC. On the other hand, if $\left|\hat{y}_{s}(t)\right|<\varepsilon_{2}$, the control law will be switched to the normal SMC-AGMC automatically when the sensor has no fault.

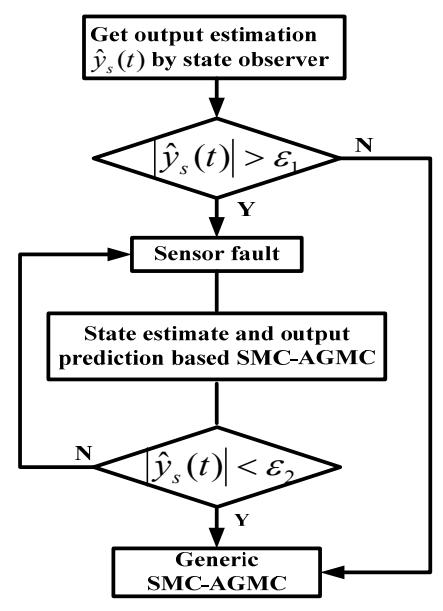

Fig. 1. The control law reconfiguration when sensor fault occurs.

\section{FAult-Tolerant Control Architecture Model}

Because the Kalman filter in traditional adaptive generic model has poor robustness to model error, the control effect is poor too. In this paper, the state observer (3) is used to estimate the system state and sensor fault online to improve the stability and reliability of the system. Also, SMC and AGMC are combined to improve the robustness. Fig. 2 shows the SMC-AGMC fault-tolerant control architecture model with the state observer.



Fig. 2. Fault-tolerant control architecture model.

The fault-tolerant control architecture model is constructed as following steps. First, the state observer (3) is used to estimate system state $x(t)$ and output $y(t)$ online. Next, get the state estimation $\hat{x}(t)$, output estimation $\hat{y}(t)$, and sensor fault estimation $\hat{y}_{s}(t)$. Then, according to estimation $\hat{y}_{s}(t)$ to judge that the sensor has fault or not. If the sensor is normal, choose the control law (7). If the sensor fault is detected, reconfigure the sensor. The estimation $\hat{x}(t)$ and $\hat{y}(t)$ are used to construct the state estimation and output prediction based 
on SMC-AGMC. $\hat{y}_{s}(t)$ is used to construct the fault detection and isolation algorithm. In this case, choose the control law (9). If the sensor returns to normal, keep the choice of the actual system output $y(t)$ and the control law (7).

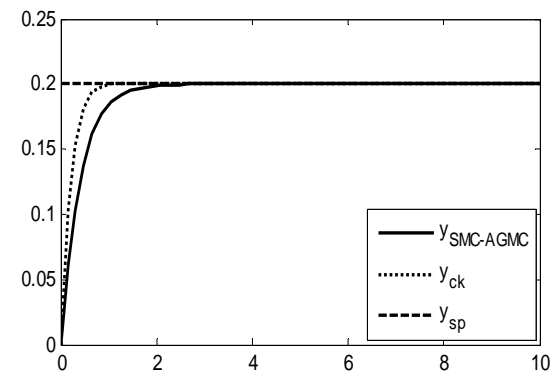

Fig. 3. Model output $y_{S M C-A G M C}$, reference output $y_{c k}$ and setting output $y_{s p}$.

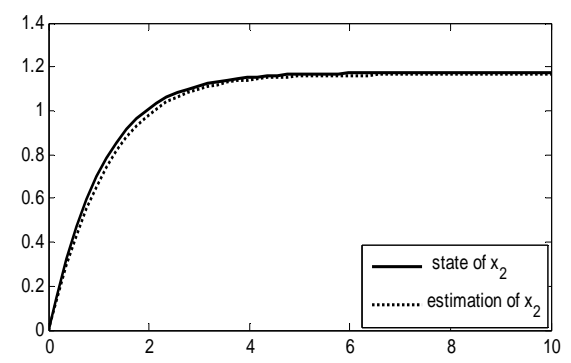

Fig. 4. Model state $x_{2}$ and estimation.

\section{Simulation Result}

Considering the T-S fuzzy descriptor model with uncertainties and double time-delays is described as follows:

$$
\begin{aligned}
& R_{i}: \text { If } \xi_{1}(t) \text { is } M_{1 i} \text { and } \xi_{\mathrm{p}}(t) \text { is } M_{p i}, \text { then } \\
& x(t)=\sum_{i=1}^{r} q_{i}(\xi(t)) \cdot\left\{\begin{array}{l}
{\left[A_{i}+\Delta A_{i}(t)\right] x(t)+\left[B_{i}+\Delta B_{i}(t)\right] u(t)} \\
+\left[A_{i d}+\Delta A_{i d}(t)\right] x(t-d) \\
+\left[B_{i d}+\Delta B_{i d}(t)\right] u(t-\tau)
\end{array}\right. \\
& y(t)=\sum_{i=1}^{r} \mu_{i}(\xi(t)) C_{i} x(t), i=1,2, \ldots, r
\end{aligned}
$$

where the parameters is taken as follows:

$$
\begin{aligned}
& q_{1}\left(x_{1}(t)\right)=1-\left(1+e^{-2 x_{1}}\right)^{-1}, q_{2}\left(x_{2}(t)\right)=\left(1+e^{-2 x_{1}}\right)^{-1}, \quad E=\left[\begin{array}{lll}
1 & 0 ; 0 & 1
\end{array}\right], \\
& A_{1}=\left[\begin{array}{lll}
0 & 2 ;-1 & 3
\end{array}\right], A_{2}=\left[\begin{array}{lll}
1 & 2 ; 0 & -1
\end{array}\right], A_{11}=\left[\begin{array}{lll}
1 & 2 ;-1 & 0.5
\end{array}\right], \\
& B_{1}=\left[\begin{array}{ll}
1 & 0
\end{array}\right]^{T}, B_{2}=\left[\begin{array}{ll}
-0.5 & 1
\end{array}\right]^{T}, A_{12}=\left[\begin{array}{lll}
-0.5 & 1 ; 1 & 0.5
\end{array}\right], B_{11}=\left[\begin{array}{ll}
0 & 1
\end{array}\right]^{T}, \\
& B_{21}=\left[\begin{array}{ll}
0 & 1
\end{array}\right]^{T}, C=\left[\begin{array}{ll}
1 & 0
\end{array}\right], E_{11}=\left[\begin{array}{ll}
0.2 & 0
\end{array}\right], E_{21}=0.1, \quad E_{31}=\left[\begin{array}{ll}
0.1 & 0
\end{array}\right], \\
& E_{41}=0.2, \quad d=0.1, \quad \tau=0.5
\end{aligned}
$$

If the output is set as $y_{s p}=0.2$, the parameters in SMC-AGMC control method are $\varepsilon_{1}=1, \varepsilon_{2}=0.2, k_{1}=5$ and $k_{2}=0.5$.

When the initial conditions are chosen as $x_{0}=\left[\begin{array}{ll}0 & 0\end{array}\right]$, the simulation results of system output and input are shown as follows:

Fig. 3 shows the system output $y$-SMCAGMC of the adaptive fault-tolerant generic model containing sliding model control, the reference model output $y_{c k}$, and the setting output $y_{s p}$. The output of system has a little deviation from the reference trajectory while it is consistent with specified output.

The system input $x_{2}$ and the state observer estimation $\hat{x}_{2}$ are shown in Fig. 4 where the trajectory of the state estimation $\hat{x}_{2}$ traces the state $x_{2}$ well. It means that the proposed adaptive fault-tolerant control structure of T-S fuzzy model is effective.

\section{CONCLUSION}

In this paper, the descriptor system containing uncertainties and time-delays is described by T-S fuzzy model. The observer is designed based on parallel distributed compensation (PDC) algorithm which can estimate system state and sensor fault directly. Thus, the fault detection system was established. Having combined SMC and AGMC, a strategy of sensor adaptive fault-tolerant generic model control is constructed. Finally, the effective control law reconfiguration logic was proposed when the sensor has fault. Also the adaptive fault-tolerant control structure was established based on sliding model control with state observer.

The results of simulation show that state-observer can estimate sensor fault, the system output is consistent with the setting output, and the control system keeps good effect. This demonstrates the proposed adaptive fault-tolerant control structure based on T-S fuzzy model was feasibility and validity. As well as, it has explicit physic meaning, simple structure, strong robustness, and easy tuning property. It is an effective fault-tolerant control strategy for a complex nonlinear control.

\section{REFERENCES}

[1] B. Liang, T. Q. Chang, and G. S. Wang, "Robust $\mathrm{H}_{\infty}$ fault-tolerant control against sensor and actuator failures for uncertain descriptor systems," Engineering, vol. 15, pp. 979-983, Dec. 2011.

[2] L. Zhang, H. Yang, and Y. W. Jing, "Fault tolerant control of uncertain time-delays systems based on T-S fuzzy models," in Proc. Chinese Control and Decision Conference, 2008, pp. 3362-3366.

[3] F. Afef and N. C. Fahmida, "A robust fault tolerant control strategy for a class of nonlinear uncertain systems," in Proc. the 2006 American Control Conference Minneapolis, 2006, pp. 5474-5482.

[4] T. Taniguchi, and M. Sugeno, "Fuzzy identification of systems and its applications to modeling and control," IEEE Transactions on Systems, Man and Cybernetics, vol. 1, pp. 116-132, Jan. 1985.

[5] B. Chen and X. P. Liu, "Reliable control design of fuzzy dynamic systems with time-varying delay," Fuzzy Sets and Systems, vol. 3, pp. 349-374, Jan. 2004.

[6] B. Y. Zhu, and Q. L. Zhang, "Robust H_o control for T - S fuzzy descriptor systems with time-delays," Electric Machines and Control, vol. 4, pp. 352-356, Apr. 2005.

[7] T. M. Guerra, A. Kruszewski, L. Vermeiren, and H. Tirmant, "Conditions of output stabilization for nonlinear models in the Takagi-Sugeno's form,” Fuzzy Sets and Systems, vol. 9, pp. 1248-1259, Jan. 2006.

[8] Z. W. Gao, X. Y. Shi, and S. X. Ding, "Fuzzy state/disturbance observer design for T-S fuzzy systems with application to sensor fault estimation," IEEE Trans. Systems Man Cybernetics, vol. 3, pp. 875-880, July 2008.

[9] P. L. Lee and G. R. Sullivan, "Generic model control," Computers and Chemical Engineering, vol. 6, pp. 573-580, June 1988.

[10] B. J. Guo, and X. M. Hua, "Generic model controller tuning for chemical processes with input saturation," Canadian Journal of Chemical Engineering, vol. 2, pp. 275-280, Dec. 2001. 
[11] P. D. Signal and P. L. Lee, "Generic model adaptive control," Chem Comm, vol. 1, pp.35-52, Apr. 1992.

[12] G. Cheng and S. X. Pan, "Adaptive sliding model control of elect-hydraulic system with nonlinear unknown parameters," Control Engineering Practice, vol. 11, pp. 1275-1284, Nov. 2008.

[13] M. C. Pai, "Observer-Based adaptive sliding mode control for nonlinear uncertain state-delayed systems," International Journal of Control, Automation and Systems, vol. 4, pp. 536-544, Apr. 2012.

[14] Z. W. Gao, X. Y. Shi, and X. D. Steven, "Fuzzy state/disturbance observer design for T-S fuzzy systems with application to sensor fault estimation," IEEE Trans. Systems Man Cybernetics, vol. 3, pp. 875-880, July 2008.

[15] T. M. Guerra, A. Kruszewski, L. Vermeiren, and H. Tirmant, "Conditions of output stabilization for nonlinear models in the Takagi-Sugeno's form," Fuzzy Sets and Systems, vol. 9, pp. 1248-1259, Jan. 2006.

[16] B. Y. Zhu, Q. L. Zhang, and S. C. Tong, "The stability criterions for fuzzy descriptor systems with time-delay," Control and Intelligent Systems, vol. 3, pp. 223-227, Jan. 2007.

[17] B. Marx, D. Koenig, and J. Ragot, "Design of observers for Takagi Sugeno descriptor systems with unknown inputs and application to fault diagnosis," IET Control Theory and Applications, vol. 1, pp. $1487-1495$, Oct. 2007.

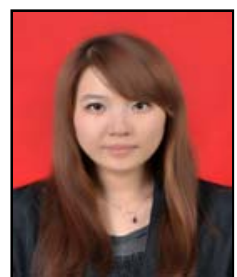

Z. J. Yu was born on May 7, 1990 in Baishan, China. Currently, she is still pursuing her master degree in the school of Yanbian University, Yanji, China.

She completed bachelor degree electronics engineering and technology, Jilin, Yanji, in the field of electronics and telecommunication, in 2013.

Her research interests include fault-tolerant control and T-S fuzzy control.



Y. N. Xu was born at Jilin Province of China. Currently, he is an associate professor of the division of electronic and communication engineering of Yanbian University, Yanji, China.

$\mathrm{He}$ received the Ph.D. degree in electrical engineering from the Chonbuk National University, Korea, in 2009.

His research interests include the in-vehicle networks and automobile electronic control. 